\title{
Acceptability, Barriers and Facilitators of Mobile Text Message Reminder System Implementation in Improving Child Vaccination: A Qualitative Study in Northwest Ethiopia
}

This article was published in the following Dove Press journal:

Journal of Multidisciplinary Healthcare

\author{
Zeleke Abebaw Mekonnen (iD) ${ }^{1,2}$ \\ Kassahun Alemu Gelaye $\mathbb{D}^{3}$ \\ Martin C Were ${ }^{4}$ \\ Binyam Tilahun' \\ 'Department of Health Informatics, \\ Institute of Public Health, College of \\ Medicine and Health Sciences, University \\ of Gondar, Gondar, Ethiopia; ${ }^{2}$ Health \\ System Directorate, Federal Ministry of \\ Health, Addis Ababa, Ethiopia; \\ ${ }^{3}$ Department of Epidemiology and \\ Biostatistics, Institute of Public Health, \\ College of Medicine and Health Sciences, \\ University of Gondar, Gondar, Ethiopia; \\ ${ }^{4}$ Department of Biomedical Informatics, \\ Vanderbilt Medical Center, Nashville, TN, \\ 37232, USA
}

Background: Mobile phone text message-based mHealth interventions have shown promise in improving health service delivery. Despite the promising findings at a small scale and few contexts, implementing new technologies as part of changes to health care services is inherently challenging. Though there is a potential to introduce mHealth initiatives to health systems of developing countries, existing evidence on the barriers and facilitators of implementation in different contexts is not adequate. Therefore, this study aimed to explore the acceptability, barriers and facilitators of implementing mobile text message reminder system for child vaccination in Ethiopia.

Methods: This study applied a phenomenological study design. The study was conducted in north-west, Ethiopia between July 28 and August 19, 2020. A total of 23 participants were purposively selected for the in-depth and key informant interviews. We used an interview guide to collect data and audio-records of interviews were transcribed verbatim. Coding was done to identify patterns and thematic analysis was conducted using ATLAS ti7 software.

Results: The findings indicated that mothers were receptive to mobile text message reminders for their child's vaccination. Low mobile phone ownership, access to mobile network, access to electricity and illiteracy among the target population were identified as barriers that would affect implementation. Confidentiality and security-related issues are not barriers to implementation of text message reminders for child vaccination service. Facilitators for implementation include stakeholder collaboration, providing orientation/training to users, and willingness to pay by clients.

Conclusion: In this study, using mobile phone text message reminders for child vaccination services are acceptable by clients. Barriers identified were related to inadequate ICT infrastructure and other technical issues. Addressing the potential barriers and leveraging the existing opportunities could optimize the implementation in resource-limited settings. Before actual implementation, program implementers should also consider providing orientation to users on the proposed mHealth program.

Keywords: acceptability, barrier, facilitator, mHealth, text message, vaccination

\section{Background}

Ensuring high vaccination coverage is a priority for public health programs recommended by the World Health Organization (WHO). ${ }^{1}$ However, vaccination coverage and timeliness have remained suboptimal in developing countries. ${ }^{2,3}$ Among the contributing factors for the sub-optimal vaccination coverage is missed attendance
Correspondence: Zeleke Abebaw Mekonnen

P.O. Box: 196, University of Gondar,

Gondar, Ethiopia

Tel +25l 913747092

Email zelekeabebaw7@gmail.com
Journal of Multidisciplinary Healthcare 2021:|4 605-6|6

submit your manuscript

Dovelress if in

http://doi.org/10.2147/1MDH.S298167 
to scheduled vaccination appointments. ${ }^{2-5}$ Currently, the immunization program of Ethiopia routinely uses vaccination cards and verbal reminders provided by health workers to remind caregivers of infants. ${ }^{6}$ The 2016 Ethiopian Demographic and Health Survey (EDHS) report found that only $46 \%$ of children age $12-23$ months were reported to have a vaccination card and interviewers were able to see a vaccination card for only $34 \%$ of children. ${ }^{7}$ Other existing reminder strategies for vaccination appointment reminders are also reported as inadequate to achieve the desired targets set at a national level. ${ }^{4}$

In recent years, the advent of technological innovations has created new ways of addressing public health challenges including health care appointment attendance. The use of mobile technologies for health service delivery is new and rapidly progressing to improve healthcare delivery including child vaccination. ${ }^{8-10}$ As mobile technology becomes more affordable and more accessible in lowincome countries, it presents more opportunities to strengthen health services. ${ }^{11-13}$

Reports indicated that $80 \%$ of the population in developing countries owned a mobile phone and in Sub-Saharan Africa more than $60 \%$ of individuals have access to a mobile phone. ${ }^{14}$ Similarly, the EDHS report in Ethiopia indicated that mobile phone ownership among urban households was $88 \%$ while it was $47.2 \%$ in rural households. ${ }^{7}$ Among the mobile phone features, short message service (SMS) remains one of the most popular forms of mobile communication and has become the most used data transfer system globally. ${ }^{8,15,16}$

Though mHealth interventions have demonstrated the potential to improve health service delivery, implementing new technologies as part of changes to health care services is inherently challenging. ${ }^{17-21}$ Evidence also indicated that the success of implementing mHealth initiatives varied in different contexts. ${ }^{18-21}$ In resource-limited settings, mHealth solutions are infrequently brought to scale or integrated into routine health service provision. ${ }^{16,22}$ Many promising technological innovations in health care are also characterized by non-adoption often explained in terms of barriers and opportunities for implementation. ${ }^{17}$

Reviews of mHealth projects in different contexts have also identified a range of challenges for implementation ranging from ICT infrastructure, digital literacy, cost implications, and security-related concerns for effective adaptation to local contexts. ${ }^{23-29}$ Another study also reported that adopting technological interventions in health care in developing countries is a challenging task and only small progress has been made to integrate digital tools into immunization programs. ${ }^{30}$ As Ethiopia aims to adapt and scale mHealth initiatives in different health programs, it is important to identify issues related with barriers and opportunities to the technology adaption. ${ }^{8,11}$ However, the existing evidence on the barriers and facilitators for effective implementation of mHealth initiatives is not adequate in Ethiopian local context. ${ }^{8,11,31}$

This study is a continuation of a randomized controlled trial study that assessed the effectiveness of mobile text message reminders on timely completion of child vaccination. The trial was a one-year follow-up study conducted in northwest Ethiopia. ${ }^{32}$ For the trial, a locally developed automated mobile text message reminder was developed and text message reminders were sent for each study participant in the intervention group one day before the due date of vaccination. The mobile text message reminders were developed and sent in Amharic and English languages for all consecutive vaccination appointments of infants. ${ }^{33}$ Therefore, this qualitative study aimed to explore the acceptability, potential barriers, and facilitators to the implementation of mobile phone text message reminder system for child vaccination in north-west, Ethiopia.

\section{Methods}

\section{Study Design and Study Period}

In this qualitative study, a phenomenological study design was applied. The study was conducted in Gondar city administration north-west, Ethiopia from July 28 to August 19, 2020.

\section{Study Population and Eligibility Criteria}

Mothers who were assigned to the intervention group for the trial study and who had functional contact addresses during the data collection period were eligible for the indepth interview. Also, mothers were eligible if they had received at least one mobile text message reminder for their infant vaccination. Besides, individuals who have been working as vaccination service provider, head of health facilities, Gondar city administration (zonal), regional, and national EPI coordinators as well as zonal (Gondar district) Ethio-Telecom manager were also included as key informants.

\section{Sample Size and Sampling Procedures}

In this study, we included a total of 23 participants (10 for IDI and 13 for KII). Though we determined the initial 
sample size, the final sample size for this study was determined based on the level of information saturation. Study participants were recruited based on purposive sampling to achieve maximum variation aimed to capture a range of experiences and demographics. For the IDI, ten mothers selected from the trial study intervention group were included. Additionally, for the KII participants were selected purposively at different levels. Accordingly, we recruited six health care workers from EPI units of health facilities and three health facility heads. Moreover, we recruited EPI coordinators at zonal, regional, and national levels. The zonal Ethio-Telecom office manager was also included to verify specific issues related to the role of Ethio-Telecom companies in the health care delivery process.

\section{Data Collection Tools and Procedures}

After reviewing relevant literature, an interview guide was developed for in-depth and key informant interviews. The English version was translated to Amharic language for data collection. The interview guide was reviewed by domain experts and refinement has been done accordingly. The interview guide was also pretested on three participants who were not included in this particular study. Based on the inputs from the pretest, the interview guide was revised for actual data collection. The interview guide included questions that address the participant's sociodemographic characteristics and acceptability of mobile text message reminders by users. It also included questions to explore barriers and facilitators to effective implementation of mobile text message-based mHealth interventions for the immunization program in Ethiopian context.

Data collection was carried out by the principal investigator and two trained data collectors. Qualitative data were collected using both audio-recorded face-to-face interviews and telephone-based interviews. Since it was not possible to meet mothers through home visits due to the COVID-19 pandemic, we recruited them through phone calls using the contact addresses they provided at the time of the trial study enrollment.

Key informant interviews with health care workers, heads of health facilities, Zonal and Regional EPI focal persons were held through face-to-face interviews. For the mothers of infants, the national EPI coordinator and the zonal Ethio-Telecom manager, the interviews were conducted through phone calls. During interviews probing questions were asked to participants to explore the issues in-depth. The interviews lasted on average of 32 minutes.

\section{Data Analysis}

Initially, audio-records of interviews were transcribed verbatim and translated into English. The translated data were cross-checked with the audio file to ensure its proper transcription and translation. The principal investigator read the translated data repeatedly to understand the concept and related meanings of the data.

We used ATLAS ti7 software for data analysis. Line by line coding was done to identify related patterns. Then codes with similar pattern were merged to identify themes from the data. During analysis, modification to existing codes and themes were made based on the information from subsequent interviews. Finally, thematic analysis was conducted to identify major themes that helped to answer the research question.

\section{Ethical Consideration}

This study was approved by the University of Gondar Institutional Review Board with ethical clearance provided (ref no: O/V/P/RCS/05/060/2018) to conduct the study. During data collection, all participants provided informed consent and the study was conducted in accordance with the Declaration of Helsinki. The participant informed consent included publication of anonymized responses. Moreover, due attention was given to not personalize any of the participant's information during data analysis, interpretation and presentation.

\section{Results}

\section{Socio-Demographic Characteristics of Study Participants}

A total of 23 participants (10 for IDI and 13 for KII) were interviewed for this study. Mothers' mean age was 28.1 years old (range of 22 to 38 years). The key informants included for this study had a mean age of 34.4 years old (range of 27 to 48 years). The key informants' work experience in their current job position ranges from six months to eighteen years [Table 1].

\section{Acceptability of Mobile Text Message Reminders}

In this study mothers who had received text message reminders were asked about their experience in using mobile text message reminders for their child's vaccination. In addition, key informants were asked about the importance of integrating mobile text message reminders for the immunization program in Ethiopia. Acceptability 
Table I Socio-Demographic Characteristics of Study Participants, 2020

\begin{tabular}{|c|c|c|c|c|}
\hline Participant ID & Age in Years & Educational Status & Current Position & Work Experience on Current Position \\
\hline PI & 48 & Degree & EPI unit focal & I year \\
\hline P2 & 29 & Degree & Health center head & 6 years \\
\hline P3 & 30 & Diploma & EPI unit focal & 2 years \\
\hline P4 & 28 & Degree & EPI unit focal & 10 years \\
\hline P5 & 34 & Degree & Health center head & 4 years \\
\hline P6 & 35 & Degree & Regional EPI coordinator & 5 years \\
\hline P7 & 33 & Degree & National EPI coordinator & 6 years \\
\hline P8 & 27 & Degree & EPI unit focal & 6 months \\
\hline P9 & 31 & Degree & EPI unit focal & 2 years \\
\hline PIO & 36 & Diploma & EPI unit focal & 3 years \\
\hline PII & 38 & Degree & Zonal Ethio-Telecom manager & 2 years \\
\hline $\mathrm{P} 12$ & 39 & Degree & Health center head & 2 years \\
\hline $\mathrm{PI} 3$ & 39 & Degree & Zonal EPI coordinator & 18 years \\
\hline $\mathrm{PI} 4$ & 38 & No formal education & Client & NA \\
\hline PI5 & 30 & Masters & Client & NA \\
\hline PI6 & 22 & Grade 9 & Client & NA \\
\hline PI7 & 25 & Grade 8 & Client & NA \\
\hline PI8 & 26 & Grade 10 & Client & NA \\
\hline PI9 & 23 & Gradel0 & Client & NA \\
\hline P20 & 27 & Diploma & Client & NA \\
\hline P2I & 29 & Diploma & Client & NA \\
\hline P22 & 30 & Diploma & Client & NA \\
\hline p23 & 31 & $10+1$ & Client & NA \\
\hline
\end{tabular}

Abbreviation: NA, not applicable.

of mobile text message reminders for child vaccination was organized into three sub-themes: importance, clarity, and timing of mobile text messages.

\section{Importance of Mobile Text Message Reminders}

The study participants reported that mobile text message reminders are important to improve child vaccination services. In this study, mothers who received mobile text message reminders were receptive towards utilizing text message-based mHealth interventions for child vaccination. They also explained the benefits of text message reminders and they felt that the text message reminders helped them to remember their appointment for child vaccination. The key informants also mentioned that mobile text message reminders are helpful to improve child vaccination service.

... Since they [text messages] remind me of my child's vaccination date, I am happy for getting those text messages which helped me to remember my child's vaccination appointment dates ... [P16, IDI, Client]

Another client also reported that;

... You know it is a good thing to get those text reminders since we may forget our child's vaccination schedule. Moreover, we might lose a child's vaccination appointment card, in this case, we will be curious on our child's 
vaccination date since those text messages can alleviate this and other related problems ... [P17, IDI, Client]

\section{Content Clarity of Mobile Text Message Reminders} Delivering a mobile text message which clients can read and understand easily is important for the effective implementation of text message-based mHealth programs. In this study, mothers who received those mobile text message reminders were comfortable with the content and clarity of text messages sent for their infant's child vaccination appointments.

I think the existing content is adequate, so it is better if you continue using it for the future. I also recommend text messages to be written in Amharic since that is the community easily understood language. [P15, IDI, Client]

... You know the current content of text messages itself is descriptive with precise information, so I can read and get its main point easily. I experience no technical challenge related to that ... [P22, IDI, Client $]$

The key informants also mentioned that the text messages should be clear and easy to understand by end-users.

I think it is better to make messages more friendly, a message which clients can understand easily, a message which clients can read easily and a message which can remind them easily. In terms of language, it is better to send messages in a language which clients better understand and can read. [P6, KII, Regional coordinator]

\section{Timing of Mobile Text Message Reminders}

To increase its chance of readability, the timing of text messages to intended users is crucial. This study revealed that mothers are comfortable receiving those mobile text messages sent before a child's vaccination due date on day-time hours.

... I am fine with the text messages sent a day before her [child's] vaccination date. Since our mobile phone might be turned off due to battery power, it is good sending the text messages a day before the vaccination appointment date during daytime hours. [P16, IDI, Client]

\section{Potential Barriers to Implementation}

Despite the huge potential in mHealth, there are potential barriers to its implementation. Subthemes were emerged to explain how potential barriers could hinder the initiation and implementation of the mHealth intervention. Infrastructure-related barriers like mobile phone access and access to electricity were reported as potential barriers during implementation. Besides, the literacy status of endusers and language barriers were mentioned as potential barriers for implementation of text message-based mHealth interventions for immunization program in Ethiopia.

\section{Infrastructure-Related Barriers}

Better network coverage and access to electricity create a greater opportunity for a wider implementation of mHealth interventions. Though there is growing expansion in the ICT infrastructure at the national level, network and electricity access or interruption were mentioned as potential barriers for scale-up of mHealth interventions. In terms of mobile phone ownership, low and inconsistent phone ownership of mothers especially in rural areas were reported as a potential barrier for future implementation.

If we plan to implement at the national level, there is a high rate of mobile access nationally. Around 60 million individuals in Ethiopia are mobile phone users and this is a big opportunity for us. We can reach millions of children using this mobile network. But still, there are challenges related to infrastructure availability and access like electricity and network, especially in rural areas. Whenever there is an expansion of network, electricity and ICT infrastructures, our chance to reach clients will be increased similarly. [P7, KII, national EPI coordinator]

One of the challenges is that most individuals do not have a mobile phone at the household level, they give their neighbors or relatives phone numbers. So, when we try to reach them through such individuals, most of the time we will not be effective since they give different reasons as they do not meet with mother or caretaker of that child. [P10, KII, EPI unit focal person]

With the given barriers in access to mobile phones and mobile networks, a key informant from Ethio-Telecom elaborated that these challenges are getting solved as time goes by, creating greater opportunities for future implementation.

... The main thing to provide such a service is to ensure whether individuals have access to mobile phones and mobile networks. Our SIM card sell rate is increasing at a significant rate. As Ethio-Telecom, we are now accessible with mobile networks in many areas, except in some remote areas. Even we are working to reach those areas through different expansion activities. If you look at the network access at the national level, $90 \%$ of the country 
has access to mobile network ... [P11,KII, Zonal Ethio-

Telecom Manager]

Moreover, the participants reported that power shortage for mobile phone charging and mobile network access could also compromise message delivery to the intended users of the program.

... since you only send a text message I do not know what measures you will take for individuals whose phone is not functional due to different problems like loss of power to charge mobile and network access. Besides that some individuals might lose or change their contact address ... [P21,IDI, Client]

In this study, establishing a reliable ICT environment and readiness of health facilities was reported as a prerequisite for the successful implementation of mHealth interventions. Regarding health facility readiness, a key informant reported that;

There is no any available resource from the health center since our health center had no income beside budget assigned from government, we cannot fulfill required materials and software unless the government allocate budget for such services. [P4,KII, EPI unit focal person]

\section{Illiteracy of Users}

Illiteracy is an issue for future implementation of text message-based mHealth interventions on the target population. One of the obstacles for end-users is a lack of digital skills to work with mHealth interventions on identifying, reading, and understanding the received text messages. It was suggested that mHealth technologies should be user-friendly and very easy to use by end-users of the program.

... You know the educational status of mothers might affect the effectiveness of this program. The majority of the community has difficulty answering or receiving calls live alone to read and understand text messages. Even when we call and try to communicate with them, sometimes they will give their phone to other individuals near them to receive voice call messages ... [P21,IDI, Client]

A key informant also supplemented that;

In a rural community, if they are not educated, they will not read messages sent to their mobile phone at all. If they do not read, they will not know what kind of message they have received even. In such a scenario, their educational status will have a serious effect. But in an urban setting, the majority of residences can read and write, so it will not be difficult for them to easily understand text messages and apply messages immediately for its intended purpose. [P3,KII, EPI unit focal person]

\section{Language Barrier}

In terms of language, study participants reported that messages should be sent in a language that clients can read and better understand, with a preference for the received information to be in the local language. Though individuals can read texts written in their local language, the language barrier could exist if text messages are written in a language they cannot read and understand. A key informant also emphasized the need to send those text messages using national and local languages for national scaleup.

... Though I have no problem receiving text messages written in English, I recommend sending text messages in local/national language that is Amharic to reach the community. Moreover sending messages in Amharic might improve its readability among illiterate mothers. [P19,IDI, Client]

At the national level, we use text messages for behavioral change communication activities. Most of the time $\mathrm{MOH}$ send messages using Amharic language and there are phones which do not detect Amharic phonetic or alphabet, this is might be one of the challenges. Even language can be a huge barrier for many individuals, for example, if we send messages using Amharic language there might be individuals who cannot read and understand Amharic though this challenge might not be as such huge. [P7, KII, National EPI coordinator]

\section{Confidentiality and Security Issues}

Building end-user trust in mHealth intervention implementation is important to increase the promotion of mHealth initiatives. Concerns regarding the confidentiality and security of personal health information residing on a mobile device were hypothesized to present as a barrier, but this was not found to be an inhibitor to use for the immunization program.

... you know, coordinators of the program informed me as they would send text message reminders for my child vaccination appointment when they recruited me to this program. Since they gave me clear information about the program initially, I had no such concerns. If anyone did not orient me about the program and as they would send 
text message reminders, I might hesitate to have trust on those received text messages .... [P19,IDI, Client]

The key informant from Ethio-Telecom also assured that confidentiality and security issues will not be a barrier for future implementation given that only authorized people to have access to the health information sent via mobile devices.

We can give $100 \%$ guarantee on ensuring the confidentiality of clients' data. For example, anyone had no authority and chance to look at text messages sent to clients except those individuals who can look at that phone personally. We only allow access to personal information only if there is an issue of national security from an authorized body. [P11, KII, Zonal Ethio-Telecom manager]

\section{Facilitators to Implementation}

Being in the era of technology is one of the major opportunities to implement this program. Moreover, there is a huge demand to support health services with technology. Facilitators for successful implementation of using mobile text messages as a reminder for child vaccination appointments are explored in three sub-themes: stakeholder collaboration, providing orientation/training, and willingness to pay for the mobile text message reminder.

\section{Stakeholders Collaboration}

Participants mentioned that multi sectoral-collaboration among different stakeholders as one of the facilitators to support effective implementation. A crucial facilitator is therefore to create collaborative platforms including key stakeholders, such as end-users, health workers, EthioTelecom, and other stakeholders in shaping the adoption process.

... I believe it demands participation from different stakeholders including the community, this should not be left for the health sector alone. So to expand this service all stakeholders whose role is demanded need to contribute based on their responsibilities. Unless all stakeholders contribute their share responsibly, we might not bring the desired change by involving only one or two of the stakeholders. If the program scaled up and all stakeholders participate and take responsibility, I believe it will improve the vaccination program ... [P1,KII, EPI unit focal person]

The respondents also explained that to help support the transitions to scale, national guidelines and regulations are needed to coordinate the objectives of mHealth initiatives with nationally defined goals and objectives, primarily before implementation. The key informants also emphasize the need for service integration where the mHealth platform could be integrated into the routine immunization program of the health facilities.

To make this program more effective there need to be a guideline on how to implement it starting from the federal level and the guideline need to answer the following questions. How to make it workable? How to implement it on the ground? Who needs to take responsibility to implement it? What system needs to be implemented and other questions? If we will try to implement such an approach, Amhara regional health bureau will have its responsibility and other government structures like the district health office, zonal health administration, and health facilities will have their responsibility too. To make this program more effective activities need to be implemented in an integrated manner. [P6, KII, Regional EPI coordinator]

Another key informant also supplemented that;

... the system needs to be integrated with health facilities routine health service delivery. For example, if this program will be managed by other bodies and send text messages for mothers to bring their child for Measles vaccination on Thursday to our health center, she will not get her desired service since we give measles vaccination only every Tuesday. So if we do not implement it in an integrated way, we might lead to client dissatisfaction and abuse ... [P12, KII, Health Center Head]

As the main stakeholder to implement this program, Ethio-Telecom is fully cooperative to collaborate with the health sector for successful implementation of mHealth programs.

... Such cooperation between telecom companies and the health sector is a global trend. So if we will have an official letter and agreement, we are very happy and interested to support the full-scale implementation in any area which demands our role ... [P11, KII, Zonal EthioTelecom Manager]

\section{Providing Orientation/Training to Users}

Proving education on how to use and implement technologies was mentioned as a facilitator in tackling barriers related to knowledge and skills. For successful implementation, participants reported that end-users need to be adequately informed and trained about the packages of the new mHealth program before actual implementation. 
... They need to give a clear description of what text messages they might get, what those messages are all about, and what they need to do when they get such messages. Mothers and caretakers need to be informed enough about the program and as they will receive text messages from health facilities and where they will bring their child for vaccination. Besides this, when we initiate this program there needs to be training and orientations to managers and health care workers ... [P6, KII, Regional EPI coordinator]

\section{Willingness to Pay for Mobile Text Message Reminders}

Full-scale implementation of mHealth interventions is not without financial implications. Hence, the service charges assumed by end-users would negatively affect mHealth uptake. Concerning the cost for the text message reminders, most of the mothers who received the text message reminders were willing to bear the cost, although few had the opinion that government should pay for the text message reminders.

... the payment for text messaging service is very minimal, it might not exceed fifty cents for one message. I do not think it will be difficult to pay such an amount of money for any individual. Since it is for my child's health I do not oppose it if you deduct it from my balance. But for individuals who give other persons' mobile phone as contact address, they might not be happy if you deduct from their balance for other individuals' child ... [P21, IDI, Client]

\section{Discussion}

This study indicated that mobile text message reminders for child vaccination are acceptable by mothers. In the study setting, the potential barriers for implementation include low mobile ownership, access to electricity, poor network connectivity, language barrier, and illiteracy of the target population. On the other hand, the facilitators for effective implementation of text message-based mHealth interventions include stakeholder engagement, willingness to pay for the service, and provision of orientation or training for users of the mHealth intervention.

In this study, using mobile text message reminders for child vaccination is acceptable by end-users. Findings in this study are consistent with previously reported studies. $^{34-38}$ The findings of text messages as the preferred type of reminder may be due to their low cost, fast delivery, and reduced interference with daily activities. In terms of content clarity of text messages, respondents reported that the text messages sent in English and Amharic languages are clear and understandable. Studies also reported that text message reminders need to be concise and delivered in a language easily understood by the community. ${ }^{34,35,39,40}$ However, with the existing technology there is a limitation of sending only 160 characters as mobile text message, which could become even less when translated into multiple languages ${ }^{41}$ affecting the content of text message reminders during development. In this study majority of the mothers were comfortable receiving the text messages one day before the due date which is consistent with findings reported in other studies. ${ }^{42-44}$

The availability of network and electricity access is very crucial for the operations of mHealth initiatives. In this study network and electricity, access were mentioned as potential barriers for effective implementation of mHealth interventions. Other studies have also shown that infrastructural challenges like poor network coverage and poor access to electricity are often major limitations to the implementation of mobile technologies for health care service delivery. ${ }^{24,37,45-48}$ This is because mHealth interventions need to have a stable mobile network and a continuous power supply to automatically send the text message reminders and recharge the mobile phones of end-users.

The illiteracy of end-users was reported as a potential barrier by some of the study participants. This finding corroborates with other studies that indicated illiteracy as an important barrier to mobile text-message-based reminders. ${ }^{34,48-54}$ This result may be explained by the fact that higher education may facilitate the use and the ability to read SMS. Evidence also showed that technical challenges would be faced by illiterate clients while using mHealth interventions. ${ }^{55}$ Illiteracy has been a problem when it comes to technical issues and as users do not appreciate device functionality and are unable to operate them, leading to failure in implementation.

Security and confidentiality issues were not reported as a barrier for implementation in this study which is consistent with another study. ${ }^{56}$ But, this finding contradicts with results reported by other studies. ${ }^{37,57,58}$ This could be justified in a way that confidentiality challenges may arise during the application of mHealth interventions for sensitive and stigmatizing health services with frequent sharing of mobile phones. In this particular study, mobile text messages were sent for vaccination service which is 
a preventive service with routine advocacy done at the community level.

In this study, stakeholder engagement was reported as a facilitator for the sustainable and effective implementation of mHealth initiatives. In another study, it was stated that key strategies to facilitate the integration of mHealth interventions in existing health systems demand collaboration between stakeholders. ${ }^{59}$ Evidence also indicated that there is a huge interest by the government of Ethiopia to integrate and implement mHealth initiatives for various health programs. ${ }^{8,11}$ Hence, multi-sectoral collaboration is very important for the success of mHealth initiatives in Ethiopia since its implementation is dependent on the collaboration of various sectors including Ethio-Telecom and non-governmental organizations.

Orientation and training for users are suggested by respondents for better implementation of the SMS-based mHealth interventions. A study from Kenya also suggested that some advocacy of the intervention and teaching of SMS content and use might be required for improved acceptability and sustainable implementation of mHealth interventions. ${ }^{47}$ Most end users may be technically challenged to use the reminder system and hence training, awareness creation, and education are highly recommended under such conditions.

Evidence indicated that one of the barriers to m-Health implementation in LMICs might be a service fee for users. ${ }^{60}$ In this study, mothers were willing to pay for the mobile text message reminders which corroborates with findings of other studies. ${ }^{34,35,39,44}$ This may demonstrate the importance placed on the vaccination of children by mothers. It is not surprising in this study that some of the mothers agreed with the idea of the government paying for the text message reminders. This may be because they are used to support the government provided free child immunization services in Ethiopia. The willingness of mothers to pay for the mobile text messages could indicate the potential of integrating the mHealth intervention costs to the health system payment modality including the community-based health insurance system.

This study has the following strengths and limitations. The study included a diverse sample of participants. To increase the transparency of the interpretation, findings were illustrated with quotations from responses of participants. This study was conducted in the urban community which may limit the transferability of the findings to different contexts. Self-reported data is subject to social desirability bias, and thus participants might have exaggerated responses. We also acknowledge that data saturation is a disputable concept and novel themes may have emerged from further interviews. ${ }^{61}$

\section{Implications for Practice}

Findings from this study could guide the future implementation of mHealth initiatives in the routine immunization program, thus optimizing their chances for success. Successful mHealth design, which takes barriers into account, may complement current practice and optimize the use of limited resources. Sustainable and effective implementation of mHealth initiatives would be daunting without providing technical support to end-users, expanding mobile networks and electricity, and health facility readiness. For mothers who cannot read mobile text messages, adding a direct call or voice message option on the mHealth system could be used as an option to improve program effectiveness in reaching the larger community. Besides, close family members could have a role to technically support mothers in this regard. For the low mobile phone ownership, alternative mitigation strategies like phone sharing among household members could be considered additionally. During implementation, taking into account the language diversity in Ethiopia is also important.

\section{Conclusion and Recommendations}

Mobile phone text message reminders for child vaccination service are acceptable by clients. Mothers who received the mobile text message reminders found it clear and understandable. ICT Infrastructure (phone ownership, network, and electricity), literacy, and languagerelated issues were considerable barriers to implementation. In this study, security and confidentiality concerns were not a barrier to the implementation of SMS-based mHealth initiatives for the EPI program in the study setting. Stakeholder's collaboration, willingness of clients to pay for the mobile text message reminders, and provision of orientation/training to end-users before actual implementation are the facilitators for implementation.

Future implementation should pay particular attention to potential barriers and leveraging the existing opportunities to optimize the implementation in resource-limited settings. Before actual implementation, program implementers should also consider providing orientation to end users on the mHealth program. Moreover, designing future strategies that could encourage collaboration between 
stakeholders and service integration to enhance large-scale implementation is recommended.

\section{Abbreviations}

eHealth, Electronic Health; EPI, Expanded Program on Immunization; FMOH, Federal Ministry of Health; ICT, Information Communication Technology; IDI, In-depth Interview; KII, Key Informant Interview; mHealth, Mobile Health; RHB, Regional Health Bureau; SIM, Subscriber Identity Module; WHO, World Health Organization.

\section{Data Sharing Statement}

The data on which these findings were developed could be available upon a reasonable request.

\section{Consent to Participate}

Study permission was acquired at all levels and informed consent was obtained from each of the study participants.

\section{Acknowledgments}

The authors would like to thank Doris Dukes Charitable Foundation (DDCF) for supporting this study through the University of Gondar. In addition, the authors are grateful for the study participants, data collectors, supervisors and stakeholders involved in this study.

\section{Author Contributions}

All authors contributed to data analysis, drafting or revising the article, have agreed on the journal to which the article will be submitted, gave final approval of the version to be published, and agree to be accountable for all aspects of the work.

\section{Funding}

This study was supported by the University of Gondar.

\section{Disclosure}

The authors report no conflicts of interest in this work.

\section{References}

1. FMOH. Ethiopia National Expanded Program on Immunization, Comprehensive Multi - Year Plan 2016-2020. Addis Ababa, Ethiopia: Federal Ministry of Health; 2015:1-115.

2. Akmatov MK, Mikolajczyk RT. Timeliness of childhood vaccinations in 31 low and middle-income countries. J Epidemiol Community Health. 2012;66(7):e14-e14. doi:10.1136/jech.2010.124651

3. Oyo-Ita A, Wiysonge CS, Oringanje C, Nwachukwu CE, Oduwole O, Meremikwu MM. Interventions for improving coverage of childhood immunisation in low- and middle-income countries. Cochrane Database Syst Rev. 2016. doi:10.1002/14651858.CD008145.pub3
4. Tilahun B, Mekonnen Z, Sharkey A, et al. What we know and don't know about the immunization program of Ethiopia: a scoping review of the literature. BMC Public Health. 2020;20(1). doi:10.1186/ s12889-020-09304-1

5. Mekonnen ZA, Gelaye KA 3, Were MC, Tilahun B. Timely completion of vaccination and its determinants among children in northwest, Ethiopia: a multilevel analysis. BMC Public Health. 2020;20(1). doi:10.1186/s12889-020-08935-8

6. FMOH, FMOH. National Expanded Program on Immunization Implementation Guideline. Revised. Edition; 2015.

7. CSA. Ethiopia Demographic and Health Survey 2016. 2016.

8. FMoH. Ethiopian national eHealth strategy. 2014. Available from: http://www.who.int/goe/policies/ethiopian_ehealth_strategy2014.pdf? ua=1. Accessed February 26, 2021.

9. Linde DS, Korsholm M, Katanga J, Rasch V, Lundh A, Andersen MS. One-way SMS and healthcare outcomes in Africa: systematic review of randomised trials with meta-analysis. PLoS One. 2019;14(6): 0217485.

10. Uddin MJ, Shamsuzzaman M, Horng L, et al. Use of mobile phones for improving vaccination coverage among children living in rural hard-to-reach areas and urban streets of Bangladesh. Vaccine. 2016;34(2):276-283. doi:10.1016/j.vaccine.2015.11.024

11. Vital Wave Consulting. mHealth in Ethiopia. Strategies for a New Framework. 2011.

12. GSMA. The mobile economy. Sub-Saharan Africa. 2017; Available from: https://www.gsma.com/mobileeconomy/sub-saharan-africa -2017/. Accessed February 26, 2021.

13. GSMA. Mobile industry impact report: sustainable development goals. September, 2016:117-123. Available from: http://www.gsma. com/betterfuture/wp-content/uploads/2016/09/_UN_SDG_Report_ FULL_R1_WEB_Singles_LOW.pdf. Accessed February 26, 2021.

14. World Bank. Mobile phone penetration. 2017. Available from: https:// blogs.worldbank.org/category/tags/mobile-phone-penetration. Accessed February 26, 2021.

15. Kharbanda EO. Helping mothers to get the message about influenza: are texts the future for increased immunization? Expert Rev Vaccines. 2015;14(3):333-335. doi:10.1586/14760584.2015.993384

16. WHO. mHealth: New Horizons for Health Through Mobile Technologies. Geneva; 2011.

17. Greenhalgh T, Wherton J, Papoutsi C, et al. Beyond adoption: a new framework for theorizing and evaluating nonadoption, abandonment, and challenges to the scale-up, spread, and sustainability of health and care technologies. J Med Internet Res. 2017;19(11):e367. doi:10.2196/jmir.8775

18. Higgs ES, Goldberg AB, Labrique AB, et al. Understanding the role of mHealth and other media interventions for behavior change to enhance child survival and development in low- and middle-income countries: an evidence review. $J$ Health Commun. 2014;19 (sup1):164-189. doi:10.1080/10810730.2014.929763

19. Deglise C, Suggs LS, Odermatt P. SMS for disease control in developing countries: a systematic review of mobile health applications. $J$ Telemed Telecare. 2012;18(5):273-281. doi:10.1258/jtt.2012.110 810

20. Wiysonge CS, Young T, Kredo T, McCaul M, Volmink J. Interventions for improving childhood vaccination coverage in lowand middle-income countries. S Afr Med J. 2015;105(11):892. doi:10.7196/SAMJ.2015.v105i11.10177

21. World Health Organization. Global Diffusion of eHealth: Making Universal Health Coverage Achievable. Report of the Third Global Survey on eHealth. 2016.

22. Campbell JI, Aturinda I, Mwesigwa E, et al. The Technology Acceptance Model for Resource-Limited Settings (TAM-RLS): a novel framework for mobile health interventions targeted to low-literacy end-users in resource-limited settings. AIDS Behav. 2017;21(11):3129-3140. doi:10.1007/s10461-0171765-y 
23. Leon N, Schneider H, Daviaud E. Applying a framework for assessing the health system challenges to scaling up mHealth in South Africa. BMC Med Inform Decis Mak. 2012;12(1). doi:10.1186/14726947-12-123

24. Aranda-Jan CB, Mohutsiwa-Dibe N, Loukanova S. Systematic review on what works, what does not work and why of implementation of mobile health (mHealth) projects in Africa. BMC Public Health. 2014;14(1). doi:10.1186/1471-2458-14-188

25. Eze GU, Adeleye OO. Enhancing routine immunization performance using innovative technology in an Urban Area of Nigeria. West Afr $J$ Med. 2015;34(1):3-10.

26. Gibson DG, Ochieng B, Kagucia EW, et al. Mobile phone-delivered reminders and incentives to improve childhood immunisation coverage and timeliness in Kenya (M-SIMU): a cluster randomised controlled trial. Lancet Glob Health. 2017;5(4):e428-e438. doi:10.1016/ S2214-109X(17)30072-4

27. CHAI. Enat Messenger for Maternal Health in Ethiopia.mHealth Compendium. 2012.

28. Ahlers-Schmidt CR, Chesser AK, Nguyen T, et al. Feasibility of a randomized controlled trial to evaluate Text Reminders for Immunization Compliance in Kids (TRICKs). Vaccine. 2012;30 (36):5305-5309. doi:10.1016/j.vaccine.2012.06.058

29. Schlumberger M, Bamoko A, Yaméogo TM, et al. Positive impact on the expanded program on immunization when sending call-back SMS through a computerized immunization register, Bobo Dioulasso (Burkina Faso). Bull Soc Pathol Exot. 2012. doi:10.1007/s13149012-0236-y

30. Tozzi AE, Gesualdo F, D’Ambrosio A, Pandolfi E, Agricola E, Lopalco P. Can digital tools be used for improving immunization programs? Front Public Health. 2016;4. doi:10.3389/fpubh.20 16.00036

31. Barkman C, Weinehall L. Policymakers and mHealth: roles and expectations, with observations from Ethiopia, Ghana and Sweden. Glob Health Action. 2017;10(sup3):1337356. doi:10.1080/165497 16.2017.1337356

32. Mekonnen ZA, Tilahun B, Alemu K, Were M. Effect of mobile phone text message reminders on improving completeness and timeliness of routine childhood vaccinations in North-West, Ethiopia: a study protocol for randomised controlled trial. BMJ Open. 2019;9 (11):e031254. doi:10.1136/bmjopen-2019-031254

33. Mekonnen ZA, Hussien FN, Tilahun BC, Gelaye KA, Mamuye AL. Development of automated text-message reminder system to improve uptake of childhood vaccination in North-West, Ethiopia. Online J Public Health Inform. 2019;11(2). doi:10.5210/ ojphi.v11i2.10244

34. Ibraheem R, Akintola M, Abdulkadir M, Adeboye M, Mohammad M. A comparative analysis of mothers' preference for specific type of phone-derived reminders for routine immunization appointments in Ilorin, Nigeria. J Med Trop. 2018;20(1):42. doi:10.4103/jomt jomt_7_18

35. Hofstetter AM, Vargas CY, Kennedy A, Kitayama K, Stockwell MS. Parental and provider preferences and concerns regarding text message reminder/recall for early childhood vaccinations. Prev Med. 2013;57(2):75-80. doi:10.1016/j.ypmed.2013.04.007

36. Smith R, Menon J, Rajeev JG, Feinberg L, Kumar RK, Banerjee A. Potential for the use of mHealth in the management of cardiovascular disease in Kerala: a qualitative study. BMJ Open. 2015;5(11): e009367-e009367. doi:10.1136/bmjopen-2015-009367

37. Krah EF, de Kruijf JG. Exploring the ambivalent evidence base of mobile health (mHealth): a systematic literature review on the use of mobile phones for the improvement of community health in Africa. Digit Health. 2016;2:205520761667926. doi:10.1177/2055207616 679264

38. Vo V, Auroy L, Sarradon-Eck A. Patients' perceptions of mHealth apps: meta-ethnographic review of qualitative studies. $J$ Med Internet Res. 2019;7(7):e13817.
39. Ibraheem RM, Akintola MA. Acceptability of reminders for immunization appointments via mobile devices by mothers in Ilorin, Nigeria: a cross-sectional study. Oman Med J. 2017;32(6):471-476. doi:10.5001/omj.2017.91

40. Kharbanda EO, Stockwell MS, Fox HW, et al. Text4health: a qualitative evaluation of parental readiness for text message immunization reminders. Am J Public Health. 2009;99(12):2176-2178. doi:10.2105/AJPH.2009.161364

41. Ahlers-Schmidt CR, Hart T, Chesser A, Paschal A, Nguyen T, Wittler RR. Content of text messaging immunization reminders: what low-income parents want to know. Patient Educ Couns. 2011;85(1):119-121. doi:10.1016/j.pec.2010.08.007

42. van Olmen J, Erwin E, García-Ulloa AC, et al. Implementation barriers for mHealth for non-communicable diseases management in low and middle income countries: a scoping review and field-based views from implementers. Welcome Open Res. 2020;5:7.

43. Balogun MR, Okafor IP, Odukoya OO, Ezeiru SS, Ogunnowo BE, Campbell PC. Access to information technology and willingness to receive text message reminders for childhood immunisation among mothers attending a tertiary facility in Lagos, Nigeria. $S$ Afr J Chem. 2012;6(3). doi:10.7196/sajch.439

44. Odinaka K, Edelu B, Achigbu K. Acceptance of mobile phone short message service for childhood immunisation reminders by Nigerian mothers. Port Harcourt Med J. 2018;12(3):127. doi:10.4103/phmj. phmj_42_17

45. Rothstein JD, Jennings L, Moorthy A, et al. Qualitative assessment of the feasibility, usability, and acceptability of a mobile client data app for community-based maternal, neonatal, and child care in Rural Ghana. Int J Telemed Appl. 2016;2016. doi:10.1155/2016/2515420

46. Ngabo F, Nguimfack J, Nwaigwe F, et al. Designing and implementing an innovative SMS-based alert system (RapidSMS-MCH) to monitor pregnancy and reduce maternal and child deaths in Rwanda. Pan Afr Med J. 2012;13.

47. Kazi AM, Carmichael JL, Hapanna GW, et al. Assessing mobile phone access and perceptions for texting-based mHealth interventions among expectant mothers and child caregivers in remote regions of Northern Kenya: a Survey-Based Descriptive Study. JMIR Public Health Surveill. 2017;3(1):e5.

48. Muller N, McMahon SA, de Neve JW, et al. Facilitators and barriers to the implementation of a mobile health wallet for pregnancy-related health care: a qualitative study of stakeholders' perceptions in Madagascar. PLoS One. 2020;15(1):e0228017.

49. Brown VB, Oluwatosin A, Ogundeji MO. Experiences, perceptions and preferences of mothers towards childhood immunization reminder/recall in Ibadan, Nigeria: a cross-sectional study. Pan Afr Med J. 2015;20. doi:10.11604/pamj.2015.20.243.6019

50. Mbuagbaw L, Bonono-Momnougui R-C, Thabane L, Kouanfack C, Smieja M, Ongolo-Zogo P. A framework for community ownership of a text messaging programme to improve adherence to antiretroviral therapy and client-provider communication: a mixed methods study. BMC Health Serv Res. 2014;14(1). doi:10.1186/1472-6963-14-441

51. Bigna JJR, Noubiap JJN, Plottel CS, Kouanfack C, Koulla-Shiro S. Barriers to the implementation of mobile phone reminders in pediatric HIV care: a pre-trial analysis of the cameroonian MORE CARE study. BMC Health Serv Res. 2014;14(1). doi:10.1186/s12913-0140523-3

52. Siedner MJ, Santorino D, Haberer JE, Bangsberg DR. Know your audience: predictors of success for a patient-centered texting app to augment linkage to HIV care in rural Uganda. J Med Internet Res. 2015;17(3):e78. doi:10.2196/jmir.3859

53. Gurupur VP, Wan TTH. Challenges in implementing mHealth interventions: a technical perspective. mHealth. 2017;3:32. doi:10.21037/ mhealth.2017.07.05

54. Nsor-Anabiah S, Udunwa MU, Malathi S. Review of the prospects and challenges of mHealth implementation in developing countries. Int J Appl Eng Res. 2019;14:12. 
55. Medhanyie AA, Little A, Yebyo H, et al. Health workers' experiences, barriers, preferences and motivating factors in using mHealth forms in Ethiopia. Hum Resour Health. 2015;13(1). doi:10.1186/ 1478-4491-13-2

56. Clark SJ, Butchart A, Kennedy A, Dombkowski KJ. Parents“ experiences with and preferences for immunization reminder/recall technologies. Pediatrics. 2011;128(5):5. doi:10.1542/peds.2011-0270

57. You WX, Comins CA, Jarrett A, et al. Facilitators and barriers to incorporating digital technologies into HIV care among cisgender female sex workers living with HIV in South Africa. mHealth. 2020;6:15. doi:10.21037/mhealth.2019.12.07

58. Powell KR. Patient-perceived facilitators of and barriers to electronic portal use: a systematic review. CIN-Comput Inform Nurs. 2017;35 (11):565-573. doi:10.1097/CIN.0000000000000377
59. Bally ELS, Cesuroglu T. Toward integration of mHealth in primary care in the Netherlands: a qualitative analysis of stakeholder perspectives. Front Public Health. 2020;7. doi:10.3389/fpubh. 2019.00407

60. Cho Y-M, Lee S, Islam SMS, Kim S-Y. Theories applied to m-health interventions for behavior change in low- and middle-income countries: a systematic review. Telemed Health. 2018;24(10):727-741. doi:10.1089/tmj.2017.0249

61. O’Reilly M, Parker N. 'Unsatisfactory Saturation': a critical exploration of the notion of saturated sample sizes in qualitative research. Qual Res. 2013;13(2):190-197. doi:10.1177/1468794112446106

\section{Publish your work in this journal}

The Journal of Multidisciplinary Healthcare is an international, peerreviewed open-access journal that aims to represent and publish research in healthcare areas delivered by practitioners of different disciplines. This includes studies and reviews conducted by multidisciplinary teams as well as research which evaluates the results or conduct of such teams or healthcare processes in general. The journal covers a very wide range of areas and welcomes submissions from practitioners at all levels, from all over the world. The manuscript management system is completely online and includes a very quick and fair peer-review system. Visit http://www.dovepress.com/testimonials. php to read real quotes from published authors. 